UT-Komaba 93-13

hepth@xxx/9310155

October 1993

\title{
Extraction of Black Hole Geometry in Exactly Quantized Two Dimensional Dilaton Gravity
}

\author{
Y. KAZAMa If AND Y. SATOH \\ Institute of Physics, University of Tokyo, \\ Komaba, Tokyo 153 Japan
}

\begin{abstract}
Based on our previous work, in which a model of two dimensional dilaton gravity of the type proposed by Callan, Giddings, Harvey and Strominger was rigorously quantized, we explicitly demonstrate how one can extract space-time geometry in exactly solvable theory of quantum gravity. In particular, we have been able to produce a prototypical configuration in which a ( smeared ) matter shock wave generates a black hole without naked sigularity.
\end{abstract}

\footnotetext{
${ }^{\dagger}$ e-mail address: kazama@tkyvax.phys.s.u-tokyo.ac.jp

${ }_{\ddagger}^{\ddagger}$ e-mail address: ysatoh@tkyvax.phys.s.u-tokyo.ac.jp
} 
Notably with the advent of a model proposed by Callan, Giddings, Harvey and Strominger (CGHS) [1], dilaton gravity in two dimensions has been widely recognized as an excellent arena in which to discuss a variety of fundamental issues in quantum gravity, especially the ones concerning the quantum properties of a black hole. Indeed we now have a large body of literature on the CGHS model and its variants, either employing semiclassical approximations [2] or attempting at exact treatments [3, 4, 5]. Semiclasscal analysis has an advantage that it is readily interpretable, but it suffers from the limitations that it is valid only for large black hole mass and for a large number of matter fields. In contrast, models amenable to exact quantization are in principle free of such limitations but in practice it is far more difficult to interpret the results in physical terms.

The purpose of this letter is to fill this vexing gap between these two approaches. Specifically, we demonstrate explicitly how one can extract space-time geometry in which a matter shock wave generates a black hole in the framework of exact quantization developed in our previous work [6] ( hereafter referred to as I). This is accomplished by fixing a gauge within the conformal gauge and computing the mean values of the matter energymomentum tensor and the (inverse) metric in a particular type of coherent physical state. Although an attempt has recently been made [7], we believe that this is the first time that one can explicitly see the emergence of space-time geometry in an exactly solvable model of quantum dilaton gravity containing matter fields. In this article, we shall describe the essence of our work. The details will be elaborated elswhere [8].

We begin with a brief review of the model and the main results obtained in I. The classical action of our model is of the CGHS form [1], given by

$$
S=\frac{1}{\gamma^{2}} \int d^{2} \xi \sqrt{-g}\left\{\mathrm{e}^{-2 \phi}\left[-4 g^{\alpha \beta} \partial_{\alpha} \phi \partial_{\beta} \phi-R_{g}+4 \lambda^{2}\right]+\sum_{i=1}^{N} \frac{1}{2} g^{\alpha \beta} \partial_{\alpha} f_{i} \partial_{\beta} f_{i}\right\},
$$

where $\phi$ is the dilaton field and $f_{i}(i=1, \ldots, N)$ are $\mathrm{N}$ massless scalar fields representing matter degrees of freedom. We take the signature of the metric to be $(+-)$ [9].

To define all the quantities unambiguously, we take our universe to be spatially periodic with period $2 \pi L$. It is then convenient to introduce coordinates $x^{\mu}=(t, \sigma)=\xi^{\mu} / L$ and require that all the fields in the action be invariant under $\sigma \rightarrow \sigma+2 \pi$. When the action is rewritten in terms of $x^{\mu}$, it retains its form except with the replacement $\lambda \rightarrow \mu \equiv \lambda L$. For physical interpretation, we will get back to the original variables $\xi^{\mu}$ and $\lambda$.

Quantization of this model enforcing conformal invariance was proposed by several authors [3, 4, 5] and we adapted the approach of Ref [4]. In this scheme, one makes 
a classical transformation $\Phi \equiv \mathrm{e}^{-2 \phi}$, and then implements an appropriate functional measure following [10]. The resulting quantum model takes a particularly simple form for $N=24$ given by

$$
\begin{aligned}
S & =S^{c l}+S^{g h}, \\
S^{c l} & =\frac{1}{\gamma^{2}} \int d^{2} x\left(-\partial_{\alpha} \Phi \partial^{\alpha} \Phi-2 \partial_{\alpha} \Phi \partial^{\alpha} \rho+4 \mu^{2} \mathrm{e}^{\Phi+2 \rho}+\frac{1}{2} \partial_{\alpha} \vec{f} \cdot \partial^{\alpha} \vec{f}\right),
\end{aligned}
$$

where $\rho$ is related to the metric and the dilaton field through $g_{\alpha \beta}=\mathrm{e}^{2 \rho-2 \omega} \eta_{\alpha \beta}$ with $2 \omega=\ln \Phi-\Phi$ and $S^{g h}$ is the usual $b-c$ ghost action. This is the model which we solved exactly in our previous work by means of a quantum canonical mapping into free fields.

From the equations of motion, the dilaton field $\Phi$ and the "Liouville" field $\rho$ can be expressed in terms of periodic free fields $\psi$ and $\chi$ as

$$
\Phi=-\chi-A B, \quad \rho=\frac{1}{2}(\psi-\Phi) .
$$

The functions $A\left(x^{+}\right)$and $B\left(x^{-}\right)$are defined by

$$
\partial_{+} A\left(x^{+}\right)=\mu \mathrm{e}^{\psi^{+/ 2}\left(x^{+}\right)}, \quad \partial_{-} B\left(x^{-}\right)=\mu \mathrm{e}^{\psi^{-/ 2}\left(x^{-}\right)},
$$

where $\psi^{ \pm / 2}\left(x^{ \pm}\right)$are the left- and right- going components of $\psi(x)$. ( The light-cone coordinates are defined as usual by $x^{ \pm}=t \pm \sigma$. ) With an abbreviation $\tilde{\gamma} \equiv \gamma / \sqrt{4 \pi}$, we write the Fourier mode expansion for $\psi$ as

$$
\psi / \tilde{\gamma}=q^{+}+p^{+}\left(x^{+}+x^{-}\right)+i \sum_{n \neq 0}\left(\left(\alpha_{n}^{+} / n\right) \mathrm{e}^{-i n x^{+}}+\left(\tilde{\alpha}_{n}^{+} / n\right) \mathrm{e}^{-i n x^{-}}\right)
$$

and similarly for $\chi / \tilde{\gamma}$ and $f^{i} / \tilde{\gamma} \equiv \phi_{f}^{i}$ with $\left(q^{+}, p^{+}, \alpha_{n}^{+}, \tilde{\alpha}_{n}^{+}\right)$replaced by $\left(q^{-}, p^{-}, \alpha_{n}^{-}, \tilde{\alpha}_{n}^{-}\right)$ and $\left(q_{f}^{i}, p_{f}^{i}, \alpha_{f, n}^{i}, \tilde{\alpha}_{f, n}^{i}\right)$ respectively. The Solution for $A\left(x^{+}\right)$which satisfies the proper boundary condition dictated by the behavior of the right hand side of the first equation in (5) is given by ( suppressing the $t$ dependence )

$$
A(\sigma)=\mu C(\alpha) \int_{0}^{2 \pi} d \sigma^{\prime} \mathrm{e}^{(1 / 2) \epsilon\left(\sigma-\sigma^{\prime}\right) \ln \alpha} \mathrm{e}^{\psi^{+/ 2}\left(\sigma^{\prime}\right)},
$$

while that for $B\left(x^{-}\right)$is similarly obtained by changing the sign of the first exponential and replacing $\psi^{+/ 2}$ by $\psi^{-/ 2}$ in (17). In these expressions, $\alpha=\exp \left(\gamma \sqrt{\pi} p^{+}\right), C(\alpha)=$ $1 /\left(\alpha^{1 / 2}-\alpha^{-1 / 2}\right)$ and $\epsilon(\sigma)$ is the usual stair-step function. Note that we must require $p^{+}$not to vanish since otherwise $C(\alpha)$ blows up. In terms of the free fields, the energymomentum tensors $T_{ \pm \pm}$take simple forms:

$$
\tilde{\gamma}^{2} T_{ \pm \pm}=\partial_{ \pm} \chi \partial_{ \pm} \psi-\partial_{ \pm}^{2} \chi+\frac{1}{2}\left(\partial_{ \pm} \vec{f}\right)^{2}
$$


It was shown in I that the mapping from the original fields $\{\Phi, \rho\}$ into the free fields $\{\psi, \chi\}$ is a quantum as well as classical canonical transformation if we assume the nonvanishing commutators among the modes of $\psi$ and $\chi$ to be $\left[q^{ \pm}, p^{\mp}\right]=i \hbar,\left[\alpha_{m}^{ \pm}, \alpha_{n}^{\mp}\right]=$ $\left[\tilde{\alpha}_{m}^{ \pm}, \tilde{\alpha}_{n}^{\mp}\right]=m \hbar \delta_{m+n, 0}$. Commutation relations for the matter fields are of the usual form.

The model so quantized is conformally invariant with the central charges $c^{d L}=2$ and $c^{f}=24$ for the dilaton-Liouville (dL) and the matter (f) sectors. $\chi$ and the product $A\left(x^{+}\right) B\left(x^{-}\right)$tranform as genuine dimension zero primary fields, while due to the presence of the background charge $\psi$ transforms anomalously as $\left[L_{m}^{d L}, \psi(x)\right]=\hbar \mathrm{e}^{i m x^{+}}\left((1 / i) \partial_{+} \psi+\right.$ $m)$.

The mathematical structure of our model is very similar to that of a bosonic string theory and all the physical states can be readily obtained through BRST analysis [11, 6]. They are of the generic form $|\Psi\rangle=\left|\Psi_{0}\right\rangle+|\Lambda\rangle$, where $\mid \Psi_{0}>$ is a special representative of a non-trivial cohomology class satisfying $L_{0}^{\text {tot }}\left|\Psi_{0}>=\bar{L}_{0}^{\text {tot }}\right| \Psi_{0}>=0$ and $\mid \Lambda>$ is the BRST trivial part. The interpretation of physical states, however, is quite different from that in a string theory: In the present model, the Virasoro level specifies the discretized energy carried by a state. As we will be most interested in configurations where the matter fields carry finite energy in the limit of large $L$, physical states at arbitrary high Virasoro levels will be of utmost importance.

To deal with such states, so called DDF representation [12] is most useful. For our model, a convenient set of spectrum-generating DDF oscillators can be constructed as 13.

$$
\tilde{A}_{-n}^{i} \equiv \mathrm{e}^{-i\left(n / \tilde{\gamma} p^{+}\right) \ln \left(\tilde{\gamma} p^{+}\right)} \int_{0}^{2 \pi} \frac{d y^{+}}{2 \pi} \mathrm{e}^{-i n \eta^{+} /\left(\tilde{\gamma} p^{+}\right)} \partial_{+} \phi_{f}^{i}\left(y^{+}\right),
$$

where

$$
\eta^{+} \equiv \ln \left(\exp \left(\tilde{\gamma} q^{+} / 2\right) A\left(x^{+}\right) / \mu\right) .
$$

$\eta^{+}$consists only of the modes of $\psi^{+/ 2}\left(x^{+}\right)$and it can be checked to be a genuine primary field of dimension 0 . Physical states can be built by applying $\tilde{A}_{-n}^{i}$ 's on a zero-mode vacuum $\mid \vec{P}>,\left(\vec{P} \equiv\left(p^{+}, p^{-}, \vec{p}_{f}\right)\right)$, satisfying the zero-energy condition $p^{+} p^{-}+(1 / 2) \vec{p}_{f}^{2}-\hbar=0$. It can be shown $[8]$ that the physical states so constructed agree precisely with the ones previously obtained in BRST formalism.

We shall now construct a physical state, which will be seen to describe a universe in which a smeared matter shock wave produces a black hole. Physical meaning of such 
an abstract state can only be extracted by looking at its response to the action of appropriate operators of physical significance and in this article we shall use the left-going energy-momentum tensor of the matter field $T^{f}\left(\xi^{+}\right)$and the inverse metric $g^{\alpha \beta}$. They are expressed in terms of the free fields as

$$
T^{f}\left(\xi^{+}\right)=\frac{1}{\tilde{\gamma}^{2}}:\left(\partial_{\xi^{+}} \vec{f}\right)^{2}:, \quad g^{\alpha \beta}=-\left(: \chi \mathrm{e}^{-\psi}:+A B \mathrm{e}^{-\psi}\right) \eta^{\alpha \beta}
$$

The normal-ordering for $T^{f}\left(\xi^{+}\right)$is the usual one while the one for : $\chi \mathrm{e}^{-\psi}$ : is defined by $: \chi \mathrm{e}^{-\psi}:=\chi \mathrm{e}^{-\psi}-\left[\chi_{a}, \mathrm{e}^{-\psi}\right]$, where $\chi_{a}$ stands for the annihiliation part of $\chi$. As all the modes of $\psi$ commute with each other, $A B \mathrm{e}^{-\psi}$ is well-defined without normal-ordering. $g^{\alpha \beta}$ so defined is finite and hermitian.

Clearly the operators we have chosen to work with are gauge dependent and hence we must specify $\mid \Lambda>$ as well as $\mid \Psi_{0}>$. There are a number of general guide lines for choosing them. As for $\left|\Psi_{0}\right\rangle$, we expect that it should be a suitable coherent state since we wish to obtain such macroscopic configuration as that of a shock wave producing a black hole. On the other hand, a simple argument on energy-momentum conservation shows that in order for the mean values to exibit non-trivial coordinate dependence we must take $\mid \Lambda>$ to be a superposition of states with various Virasoro weights.

Accordingly, we have chosen our physical state to be of the following form. First, the gauge part $\mid \Lambda>$ is chosen to be of a simple form

$$
\left|\Lambda>=\frac{1}{\hbar^{2}}\left(d b_{-1}+\bar{d} \bar{b}_{-1}\right)\right| \Omega>
$$

where $b_{-1}$ and $\bar{b}_{-1}$ are, respectively, the left- and right-going anti-ghost oscillator at level one. $\mid \Omega>$ is a superposition of zero-mode states of the form

$$
\begin{aligned}
\mid \Omega>\equiv & \sum_{k=-\infty}^{\infty} \omega_{k} \sum_{l= \pm 1,0} \mid \tilde{P}(k, l)> \\
\mid \tilde{P}(k, l)>\equiv & e^{-i c p^{+} / \hbar \gamma} \gamma^{2} \int_{-\infty}^{\infty} d p^{+} \int_{-\infty}^{\infty} d p^{-} W\left(p^{+}\right) \mid p^{+}, p^{-}(k, l), \vec{p}_{f}> \\
& \times \delta\left(p^{-}-\frac{1}{p^{+}}\left(\hbar-\frac{1}{2} p_{f}^{2}\right)\right) \\
p^{-}(k, l) \equiv & p^{-}-\frac{\hbar}{p^{+}} k-i \hbar \tilde{\gamma} l
\end{aligned}
$$

where $\omega_{k}$ are a set of real coefficients. In $\mid \tilde{P}(k, l)>$, smearing with an appropriate real weight $W\left(p^{+}\right)$, to be specified later, is introduced to make the mean value of the operator $q^{-}$well-defined, which appears in $<g^{\alpha \beta}>$. Further a BRST invariant phase factor in 
front produces a coordinate-independent contribution in $\left\langle g^{\alpha \beta}\right\rangle$ and the constant $c$ will be adjusted to cancel certain unwanted terms. Summation over states with shifted $p^{-}$ zero-modes in (13) is necessary for obtaining non-trivial expectation values.

As for the BRST non-trivial part $\mid \Psi_{0}>$, we have taken it to be

$$
\begin{aligned}
\mid \Psi_{0}> & \equiv e^{G} \mid \tilde{P}>, \quad G \equiv \frac{1}{\hbar} \sum_{n \geq 1} \frac{\tilde{\nu}_{n}}{n} \tilde{A}_{-n}, \\
\tilde{\nu}_{n} & =\nu_{n} \mathrm{e}^{i n x_{0}^{+}} \quad\left(\nu_{n}, x_{0}^{+}: \text {real constants }\right),
\end{aligned}
$$

where $|\tilde{P}>\equiv| \tilde{P}(0,0)>$ defined in (14). It is easy to see that $L_{0}^{\text {tot }}\left|\tilde{P}>=\bar{L}_{0}^{\text {tot }}\right| \tilde{P}>=0$ holds. For simplicity, we consider a coherent state in which only one kind of matter field is excited, and thus omit the superscript $i$ on $\tilde{A}_{-n}$. The phase factor in $\tilde{\nu}_{n}$ will produce a shift $x^{+} \rightarrow x^{+}-x_{0}^{+}$in certain terms and will specify where a matter shock wave will traverse.

We are now ready to describe the computations of the mean values. After a simple calculation, the mean value of a hermitian operator $\mathcal{O}$ without ghosts can be reduced to the form

$$
\begin{aligned}
<\Psi|\mathcal{O}| \Psi>= & <\Psi_{0}|\mathcal{O}| \Psi_{0}> \\
& +(1 / \hbar)<\Omega\left|\left[\mathcal{L}_{+}, \mathcal{O}\right]\right| \Psi_{0}>+(1 / \hbar)<\Psi_{0}\left|\left[\mathcal{O}, \mathcal{L}_{-}\right]\right| \Omega>\quad(18) \\
& +\left(1 / \hbar^{2}\right)<\Omega\left|\mathcal{O}\left[\mathcal{L}_{+}, \mathcal{L}_{-}\right]\right| \Omega>+\left(1 / \hbar^{2}\right)<\Omega\left|\left[\left[\mathcal{L}_{+}, \mathcal{O}\right], \mathcal{L}_{-}\right]\right| \Omega>
\end{aligned}
$$

where $\mathcal{L}_{-} \equiv L_{-1}^{\text {tot }}+\bar{L}_{-1}^{\text {tot }}$ and $\mathcal{L}_{+} \equiv L_{1}^{\text {tot }}+\bar{L}_{1}^{\text {tot }}$. The rest of the calculations, although tedious, can be performed without approximation in a straightforward way [14]. The exact results are unfortunately long and involved, and we shall display them elswhere [8]. Here instead, we present the most interesting limit, namely when the (parameter) size of the universe, $L$, becomes very large. In this limit, as the discrete energy levels tend to become continuous, we can replace certain infinite sums by integrals and the results simplify considerably.

It turns out that the following choice of parameters will produce a shock-wave-blackhole configuration:

$$
\begin{aligned}
\nu_{n} & =\nu(L u)=\nu u^{-1 / 2} \mathrm{e}^{-a u^{2}}, \quad p_{f}=0, \\
\omega_{n} & =\omega(L u)=-\omega /(L u) \quad(n \neq 0, \omega<0), \\
W\left(p^{+}\right) & =p^{+} \mathrm{e}^{-\alpha\left(p^{+}-p_{0}^{+}\right)^{2} / 2},
\end{aligned}
$$


where we have introduced a quantity $u \equiv n / L$, to be used as an integration variable, and $\nu, p_{0}^{+}, \omega$ and $\omega_{0}$ are constants. For these parameters, large $L$ limit of the mean values become

$$
\begin{aligned}
<T^{f}\left(\xi^{+}\right)> & \stackrel{L \rightarrow \infty}{\sim} \omega \nu^{2} I_{T}\left(\xi^{+}-\xi_{0}^{+}\right)<\tilde{P} \mid \tilde{P}> \\
<g^{-1}> & \equiv-<:(\chi+A B) \mathrm{e}^{-\psi}:> \\
& \stackrel{L \rightarrow \infty}{\sim}\left(-K_{g}\left(\xi^{+}\right)-\lambda_{g}^{2} \xi^{+} \xi^{-}\right)<\tilde{P} \mid \tilde{P}> \\
K_{g}\left(\xi^{+}\right) & =c_{K} \xi^{+}+d_{K}-\tilde{\gamma}^{2} \omega \nu^{2} I_{\chi}\left(\xi^{+}-\xi_{0}^{+}\right),
\end{aligned}
$$

where $I_{T}(\xi)$ and $I_{\chi}(\xi)$ are integrals of the form

$$
\begin{aligned}
I_{\chi}(\xi) & =\int_{1 / L}^{\infty} d u \frac{\cos u \xi}{u^{2}} \int_{0}^{u} d v[v(u-v)]^{-1 / 2} \mathrm{e}^{-a\left(v^{2}+(u-v)^{2}\right)} \\
I_{T}(\xi) & =-\partial_{\xi}^{2} I_{\chi}(\xi) .
\end{aligned}
$$

$\lambda_{g}^{2}$ is a certain constant, proportional to $\lambda^{2}$, which is a function of the parameters $M, \omega$, $\omega_{0}, \alpha$ and $p_{0}^{+}$, and the norm $<\tilde{P} \mid \tilde{P}>$ is a function of $\alpha$ and $p_{0}^{+}$times a $\delta$-function $\delta^{25}(0)$. We shall ignore this common factor, which can be made finite by additional smearing. The term of the form $-\lambda_{g}^{2} \xi^{+} \xi^{-}$arises entirely from the pure gauge part of $\left\langle A B \mathrm{e}^{-\psi}\right\rangle$, after a suitable adjustment of $\omega_{0}$, and it describes the so called linear dilaton vacuum when the matter field vanishes. The fact that our simple choice of $\mid \Lambda>$ produces precisely such a vacuum configuration is quite remarkable. In $K_{g}$, the constants $c_{K}$ and $d_{K}$ can be made arbitrary by adjusting $p_{0}^{+}, \alpha$ in $W\left(p^{+}\right)$and the constant $c$ introduced in the definitions of $\mid \tilde{P}(k, l)>$ and $\mid \tilde{P}>$.

By a change of variable, the integrals $I_{T}$ and $I_{\chi}$ can be brought to the following forms:

$$
\begin{aligned}
I_{T}= & \sqrt{\frac{2 \pi}{a}} \int_{0}^{1} d x\left(1-x^{2}\right)^{-1 / 2}\left(1+x^{2}\right)^{-1 / 2} \mathrm{e}^{-\xi^{2} /\left(2 a\left(1+x^{2}\right)\right)}, \\
I_{\chi}=\quad L \pi & -\sqrt{2 \pi a} \int_{0}^{1} d x\left(1-x^{2}\right)^{-1 / 2}\left(1+x^{2}\right)^{1 / 2} \mathrm{e}^{-\xi^{2} /\left(2 a\left(1+x^{2}\right)\right)} \\
& \quad-\sqrt{\frac{2 \pi}{a}} \xi^{2} \int_{0}^{1} d x\left(1-x^{2}\right)^{-1 / 2}\left(1+x^{2}\right)^{-1 / 2}{ }_{1} F_{1}\left(\frac{1}{2} ; \frac{3}{2} ;-\frac{\xi^{2}}{2 a\left(1+x^{2}\right)}\right) \\
& \stackrel{\xi \rightarrow \infty}{\sim} L \pi-\frac{\pi^{2}}{2}|\xi|,
\end{aligned}
$$

where ${ }_{1} F_{1}(a ; b ; z)$ is the confluent hypergeometric function. It is evident from (27) that $<$ $T^{f}\left(\xi^{+}\right)>$is very nearly a Gaussian peaked around $\xi_{0}^{+}$and as the parameter $a$ approaches 0 it becomes a $\delta$-function. Thus it precisely describes a (smeared) shock wave of left-going 
matter energy density discussed in [1]. As for $I_{\chi}\left(\xi^{+}-\xi_{0}^{+}\right)$, it is important to note that the asymptotic behavior for large $\left|\xi^{+}-\xi_{0}^{+}\right|$is linear as is seen in (29). In fact a numerical evaluation shows that for small $a$ this asymptotic form is accurate even for small $\left|\xi^{+}-\xi_{0}^{+}\right|$ down to $\sim \sqrt{a}$. Thus we can make $\left\langle g^{-1}>\right.$ behave very much like the CGHS case by adjusting the term $c_{K} \xi^{+}+d_{K}$ in $K_{g}$ to cancel this linear portion for the range $\xi^{+}<\xi_{0}^{+}$ and to remove the constant $L \pi . K_{g}\left(\xi^{+}\right)$then becomes

$$
K_{g}\left(\xi^{+}\right)=\tilde{\gamma}^{2} \omega \nu^{2}\left(\frac{\pi^{2}}{2}\left(\xi^{+}-\xi_{0}^{+}\right)-I_{\chi}\left(\xi^{+}-\xi_{0}^{+}\right)+L \pi\right),
$$

which behaves like $\left(\pi^{2} / 2\right) \tilde{\gamma}^{2} \omega \nu^{2} \theta\left(\xi^{+}-\xi_{0}^{+}\right)$for $\left|\xi^{+}-\xi_{0}^{+}\right|>>\sqrt{a}$. In this way we obtain a smeared version of the CGHS black hole. In Fig. 1, we show the line of curvature singularity for our configuration obtained numerically. We clearly see that a black hole without a naked singularity is formed and to the left of the line $\xi^{+}=\xi_{0}^{+}$the space-time quickly becomes the linear dilaton vacuum configuration for $\sqrt{a}<<\xi_{0}^{+}$. Accordingly, for small enough $a$, the black hole mass takes exactly the CGHS form, which in our normalization is given by $\left(\pi^{2} / 2\right) \lambda_{g} \omega \nu^{2} \xi_{0}^{+}$.

By using an exactly solvable model of CGHS type, we have shown explicitly how one can reproduce a black hole geometry from an exact yet abstract physical state in quantum theory of gravity. Although the model employed is but a toy model in $1+1$ dimensions, we believe that it is quite significant to be able to discuss one of the important issues in quantum gravity in a concrete and unambiguous manner. Furthermore, the point of view and the procedures developed in this work should find wide applications in other models of quantum gravity and possibly in quantum cosmology.

We would like to thank our colleagues, especially S. Hirano and H. Ishikawa, for discussions. The research of Y.K. is supported in part by the Grant-in-Aid for Scientific Research (No.04640283) and Grant-in-Aid for scientific Research for Priority Areas (No. 05230011) from the Ministry of Education,Science and Culture. 


\section{References}

[1] C.G. Callan, S.B. Giddings, J.A. Harvey and A. Strominger, Phys. Rev. D 45, R1005 (1992).

[2] Early references are : J.G. Russo, L. Susskind and L. Thorlacius, Phys. Lett. B 292, 13 (1992); T. Banks, A. Dabholkar, M. Douglas and M. O'Loughlin, Phys. Rev. D 45, 3607 (1992); S. Hawking, Phys. Rev. Lett. 69, 406 (1992). For further references, see [8].

[3] S.P. de Alwis, Phys. Lett. B 289, 278 (1992); A. Bilal and C.G. Callan, Nucl. Phys. B394,73 (1993).

[4] K. Hamada and A. Tsuchiya, Int. J. Mod. Phys. A ( to be published ).

[5] E. Verlinde and H. Verlinde, Nucl. Phys. B406, 43 (1993); K. Schoutens, E. Verlinde and H. Verlinde, Phys. Rev. D 48, 2690 (1993).

[6] S. Hirano, Y. Kazama and Y. Satoh, Phys. Rev. D 481687 (1993).

[7] S.P. de Alwis, University of Cololado Report No. COLO-HEP-318 ( unpublished ).

[8] Y. Kazama and Y. Satoh, University of Tokyo, Komaba Report No. UT-Komaba 93-19 ( unpublished).

[9] Compared with the form we adopted in I, the sign of the terms in the bracket [ ] are reversed to conform to original CGHS action. This however results only in a reverse of sign for $\chi$ in Eq.(4).

[10] F. David, Mod. Phys. Lett. A3, 1651 (1988); J. Distler and H. Kawai, Nucl. Phys. B321, 509 (1989).

[11] B.H. Lian and G.J. Zuckerman, Phys. Lett. B 254, 417 (1991); P. Bouwknegt, J. McCarthy, and K. Pilch, Commun. Math. Phys. 145, 541 (1992).

[12] E. Del Guidice, P. Di Vecchia and S. Fubini, Ann. Phys. 70, 378 (1972).

[13] The oscillators are intimately related to those introduced in [5].

[14] There is a technicality in the definition of the inner product for the zero-mode sector, but this can be settled by demanding that $\left\langle T^{f}>\right.$ and $g^{\alpha \beta}$ be real. For details, see [8]. 


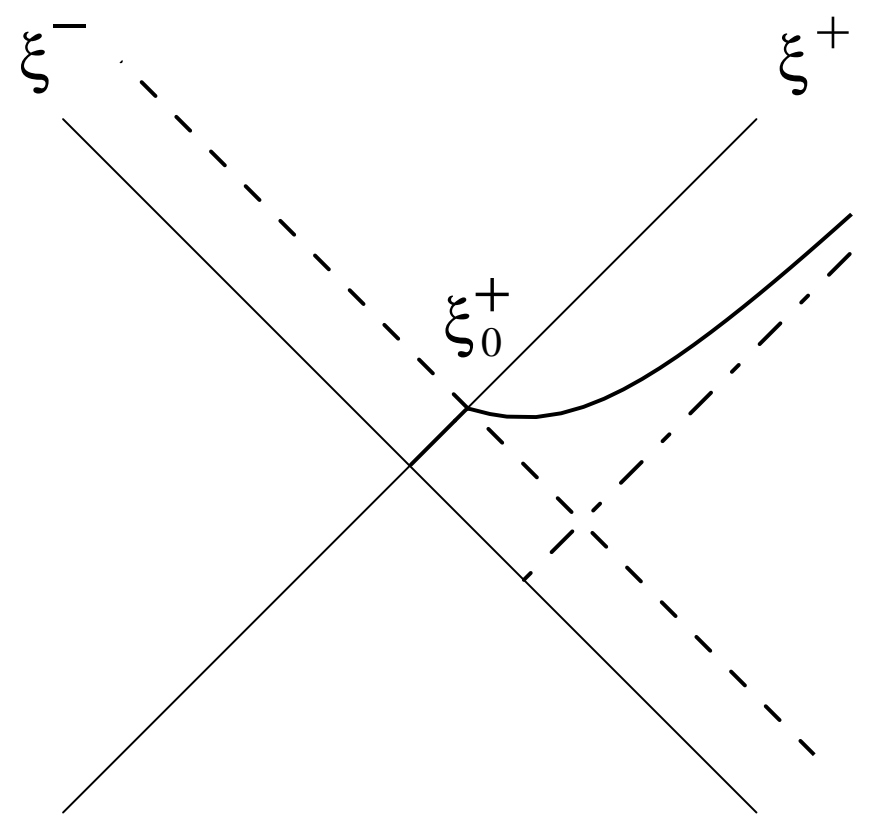

Fig.1 : The line of curvature singurarity ( solid line ) produced by a left-going smeared shock wave along $\xi^{+}=\xi_{0}^{+}$( dotted line ). The dot-dashed line represents the event horizon and the space-time quickly approaches the linear dilaton vacuum to the left of $\xi^{+}=\xi_{0}^{+}$. 